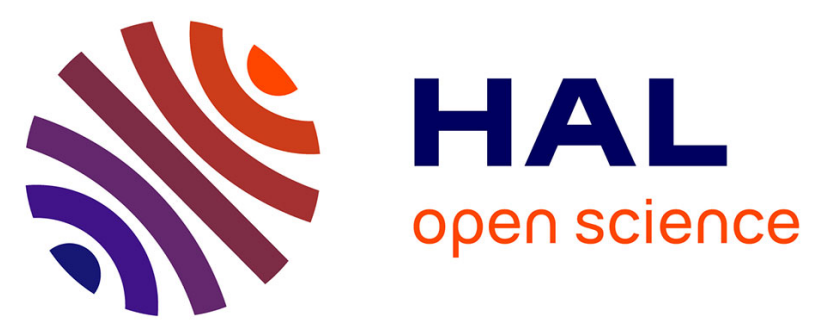

\title{
Using Schumann resonance measurements for constraining the water abundance on the giant planets - Implications for the solar system's formation
}

Fernando Simões, Robert Pfaff, Michel Hamelin, Jeffrey Klenzing, Henry Freudenreich, Christian Béghin, Jean-Jacques Berthelier, Kenneth Bromund, Rejean Grard, Jean-Pierre Lebreton, et al.

\section{To cite this version:}

Fernando Simões, Robert Pfaff, Michel Hamelin, Jeffrey Klenzing, Henry Freudenreich, et al.. Using Schumann resonance measurements for constraining the water abundance on the giant planets - Implications for the solar system's formation. The Astrophysical Journal, 2012, 750 (1), pp.85. 10.1088/0004-637X/750/1/85 . hal-00692138

\section{HAL Id: hal-00692138 https://hal.science/hal-00692138}

Submitted on 12 Mar 2015

HAL is a multi-disciplinary open access archive for the deposit and dissemination of scientific research documents, whether they are published or not. The documents may come from teaching and research institutions in France or abroad, or from public or private research centers.
L'archive ouverte pluridisciplinaire HAL, est destinée au dépôt et à la diffusion de documents scientifiques de niveau recherche, publiés ou non, émanant des établissements d'enseignement et de recherche français ou étrangers, des laboratoires publics ou privés. 


\title{
USING SCHUMANN RESONANCE MEASUREMENTS FOR CONSTRAINING THE WATER ABUNDANCE ON THE GIANT PLANETS—IMPLICATIONS FOR THE SOLAR SYSTEM'S FORMATION
}

\author{
Fernando Simões ${ }^{1}$, Robert Pfaff ${ }^{1}$, Michel Hamelin $^{2}$, Jeffrey Klenzing ${ }^{1}$, Henry Freudenreich $^{1}$, Christian Béghin ${ }^{3}$, \\ Jean-Jacques Berthelier ${ }^{2}$, Kenneth Bromund ${ }^{1}$, Rejean Grard ${ }^{4}$, Jean-Pierre Lebreton ${ }^{3,5}$, Steven Martin ${ }^{1}$, \\ Douglas Rowland ${ }^{1}$, Davis Sentman ${ }^{6,9}$, Yukihiro TaKahashi ${ }^{7}$, and Yoav YaIR ${ }^{8}$ \\ ${ }^{1}$ NASA/GSFC, Heliophysics Science Division, Space Weather Laboratory (Code 674), Greenbelt, MD, USA \\ ${ }^{2}$ LATMOS/IPSL, UPMC, Paris, France \\ ${ }^{3}$ LPC2E, CNRS/Université d'Orléans, France \\ ${ }^{4}$ ESA/ESTEC, Research Scientific Support Department, Noordwijk, The Netherlands \\ ${ }^{5}$ LESIA, Observatoire de Paris-Meudon, France \\ ${ }^{6}$ Institute of Geophysics, University of Alaska Fairbanks, Fairbanks, AK, USA \\ ${ }^{7}$ Department of Geophysics, Tohoku University, Sendai, Japan \\ ${ }^{8}$ Department Life Natural Sciences, Open University of Israel, Raanana, Israel \\ Received 2011 December 12; accepted 2012 February 26; published 2012 April 17
}

\begin{abstract}
The formation and evolution of the solar system is closely related to the abundance of volatiles, namely water, ammonia, and methane in the protoplanetary disk. Accurate measurement of volatiles in the solar system is therefore important for understanding not only the nebular hypothesis and origin of life but also planetary cosmogony as a whole. In this work, we propose a new remote sensing technique to infer the outer planets' water content by measuring Tremendously and Extremely Low Frequency (TLF-ELF) electromagnetic wave characteristics (Schumann resonances) excited by lightning in their gaseous envelopes. Schumann resonance detection can be potentially used for constraining the uncertainty of volatiles of the giant planets, mainly Uranus and Neptune, because such TLF-ELF wave signatures are closely related to the electric conductivity profile and water content.
\end{abstract}

Key words: planets and satellites: composition - planets and satellites: formation - planets and satellites: physical evolution - protoplanetary disks - space vehicles: instruments - waves

Online-only material: color figures

\section{INTRODUCTION}

The nebular hypothesis is the prevailing model to explain the formation and evolution of the solar system; specifically, the Solar Nebular Model receives most attention and, to some extent, is able to explain several characteristics of the solar system planets, namely distribution and migration, and the composition of the initial protoplanetary disk and subsequent accretion processes (e.g., Tsiganis et al. 2005). According to theory, the accretion processes induce formation of silicates and grains of ice and dust that eventually coagulate in small planetesimals and planetary embryos. Detailed analyses of these processes are not the aim of this work; thorough, comprehensive descriptions can be found elsewhere (e.g., Benz et al. 2000; Kallenbach et al. 2003). Nonetheless, it is important to mention that the water vapor and ice contents in the gaseous giants, and consequently in the protoplanetary disk volatile inventory, remain largely unknown. Measurements of the water content in the atmosphere of Jupiter and Saturn have been made by various spacecraft (Mahaffy et al. 2000; Baines et al. 2009), but generalization to the entire fluid envelope of the two planets is not possible. Because of limited in situ measurements, even the accuracy of the Jovian planets (Jupiter and Saturn) aeronomy models cannot be validated, and the water content in Uranus and Neptune is still more uncertain. Since most volatiles in the core of the primordial solar nebula are dissociated or diffused toward the outer regions during the accretion process due to temperature increase, accurate estimates of water in the giant planets and beyond would be valuable to assess volatile inventories in the

\footnotetext{
9 Deceased
}

protoplanetary disk. For example, bombardment of the inner planets by comets and asteroids originating from the outer solar system delivers water and other volatiles; terrestrial water originates, substantially, from cometary bombardment, possibly including the building blocks of life (e.g., Encrenaz 2008; Cooper et al. 2001).

Constraining initial parameterization of the protoplanetary disk is important for a better understanding of the solar system origin and evolution. For example, the distribution of rocky, icy, and gaseous bodies resulting from the protosolar nebula is linked to volatiles' abundance and to the location of the "snow line". The snow line, also known as ice or frost line, establishes the boundary in the protoplanetary disk beyond which hydrogenated molecules, namely water, methane, and ammonia, were cool enough $(\sim 150 \mathrm{~K})$ to condense and form ice grains. During the early stages of the solar system formation, the snow line was presumably located several AU from the protosun, separating the inner rocky (metals and silicates) and outer icy/gaseous (hydrogen, helium, and ices) regions (e.g., Lodders 2004).

Since the ionization energy of helium is significantly higher than that of molecular hydrogen ( 25 versus $15 \mathrm{eV}$ ), electrical conductivity of the interior of the giant planets is mainly due to hydrogen and driven by thermodynamic parameters such as temperature, pressure, and density as a function of depth. Several processes contribute to increase the electrical conductivity depending on distribution and nature of impurities. The ionization energy of water, methane, and ammonia is about 12.6, 12.6, and $10.1 \mathrm{eV}$, providing a direct contribution to conductivity increase. In addition to a stoichiometric contribution, composition also plays indirect roles in conductivity, mainly in the atmosphere, as a consequence of enhancement of aerosol-cloud interactions, 
electrophilic species chemistry, phase change, droplet formation, ion attachment, etc. Although the reaction mechanisms in the environment of giant planets are markedly different from those taking place on Earth, the dielectric properties of water, for example, are remarkably diverse in the solid, liquid, and gas phases (e.g., Petrenko \& Whitworth 2002) and can drive nucleation and clustering, as well as condensation and freezing, thus modifying charged particles mobility and recombination rates. Complementary information on volatiles in the gas giants, namely Uranus and Neptune, can be found in the work by Liu (2006). Effects of aerosols and water vapor in atmospheric conductivity and electromagnetic wave propagation in the atmosphere and ionosphere of Earth, namely in the ELF range, can be found elsewhere (Simões et al. 2011a and references therein).

Electromagnetic waves are able to penetrate into the shallow interior of gaseous planets and respond to the depth-dependent electrical conductivity of the atmosphere. This is set by the local thermodynamic state at any depth within the interior of the planet. In the case of the Earth, the surface and ionosphere form a closed cavity where electromagnetic waves can propagate. The closed cavity supports a set of normal electromagnetic modes with characteristics that depend on the physical dimensions of the cavity. When an impulsive electrical current such as lightning occurs within the cavity, the normal modes are excited to form the Schumann resonance spectrum. The Schumann resonance phenomenon has been extensively used to investigate the lightning-thunderstorm connection. Schumann resonances have been conjectured to be excited in planetary environments that possess ionosphere and show evidence for electrical activity, from Venus to Neptune, as well as Titan, the largest moon of Saturn with a thick atmosphere (Simões et al. 2008c). Unlike Venus, Mars, and Titan, where atmospheric electric discharging phenomena remain uncertain, excitation of Tremendously and Extremely Low Frequency (TLF-ELF) ${ }^{10}$ electromagnetic normal modes in the atmospheres of the outer planets is thought to be highly probable. For Jupiter and Saturn, in particular, lightning activity is certain because both very low frequency (VLF) and optical signatures attributed to lightning have been detected by various spacecraft (see Yair et al. 2008 for a review). The normal mode frequencies of the Schumann resonances are related to cavity radius and medium conductivity, which in turn is dependent on the water vapor and ice abundances in the fluid envelope (Sentman 1990b; Liu 2006; Simões et al. 2008a). Detection of the Schumann resonances and a study of their properties could therefore indirectly yield the water content in the shallow interiors of these planets by way of its effects on the conductivity profile.

Recent detection of the terrestrial Schumann resonances from orbit by the Communications/Navigation Outage Forecasting System (C/NOFS) satellite (Simões et al. 2011b) unveils new capabilities for the investigation of planetary atmospheric electricity in other planets from orbit. Previously, Schumann resonance assessments required descent probes, balloons, or landers, but the $C / N O F S$ results provide an original, remote sensing technique for TLF-ELF wave detection onboard orbiters; measurements in orbit are generally more versatile than

\footnotetext{
10 The nomenclature Ultra Low Frequency (ULF) is often used in ionospheric and magnetospheric sciences to designate frequencies below $3 \mathrm{~Hz}$. For the sake of clarity and since this work is mostly related to electromagnetic wave propagation, we use the acronym TLF-ELF to define the frequency range $0.3-30 \mathrm{~Hz}$, following the high frequency radio band classification analogy of the International Telecommunication Union.
}

in situ measurements. Complementary information on the usefulness of Schumann resonance remote sensing for atmospheric electricity investigations can be found in a review by Aplin et al. (2008).

In the following, after a brief theoretical description of the phenomenon, we examine the suitability of using Schumann resonances for determining the water/ice content in the fluid envelopes of the outer planets and, consequently, proving constraint on the volatile inventory of the protosolar nebula, hence providing new constraints for solar system formation models.

\section{SCHUMANN RESONANCE THEORY}

The propagation of low frequency electromagnetic waves within the cavity formed by two, highly conductive, concentric, spherical shells, such as those formed by the surface and the ionosphere of Earth, was first studied by Schumann (1952), and the resonance signatures of the cavity subsequently were observed in ELF spectra by Balser and Wagner (1960). Such a closed cavity supports both electric and magnetic normal modes. The lowest frequency of these modes is the transversemagnetic mode of order zero $\left(\mathrm{TM}_{0}\right)$ also sometimes called the transverse-electromagnetic (TEM) mode. These normal modes have an electric polarization that is radial, and a magnetic polarization that is perpendicular to the electric field and tangent to the surface of the planet. The modes may be excited by impulsive current sources within the cavity that, when observed as banded spectra, are known as the Schumann resonances. This phenomenon has been extensively used in atmospheric electricity investigations on Earth. Figure 1 presents a sketch of the Schumann resonance phenomenon in planetary cavities. On Earth, anisotropy due to the geomagnetic field allows for ELF wave leakage through the ionosphere (dashed line), a result also plausible in planetary environments that possess a significant magnetic field. The diagram on the right-hand side illustrates the major characteristics of the cavity, including the domain of our numerical model. For the sake of clarity, we define the interior of the giant planets as the region where the pressure is larger than 1 bar; this reference level also determines the radius of the planet. The gaseous envelope refers to the gas component of the interior, the atmosphere, and the ionosphere. The inner boundary of the numerical model is located near the solid or liquid interface. The outer boundary is placed in the ionosphere or magnetosphere; the latter is useful for investigating cavity leakage, namely computing the electric field variation with distance.

The normal mode frequencies (eigenfrequencies) of order $n$, $f_{n}$, of a lossless, thin spherical cavity can be computed from (Schumann 1952):

$$
f_{n}=\frac{c}{2 \pi R} \sqrt{n(n+1)},
$$

where $c$ is the velocity of light in vacuum, $R$ the radius of the cavity (planet), and $n=1,2,3, \ldots$ the corresponding order of the eigenmode. Taking into account the cavity thickness and medium losses, a more accurate approximation of the eigenfrequencies yields

$$
f_{n} \approx \frac{c}{2 \pi R}\left(n(n+1) \frac{1-\frac{h}{R}}{\varepsilon_{r}\left(1+i \frac{\sigma}{\varepsilon_{r} \varepsilon_{o} 2 \pi f_{n}}\right)}\right)^{1 / 2},
$$




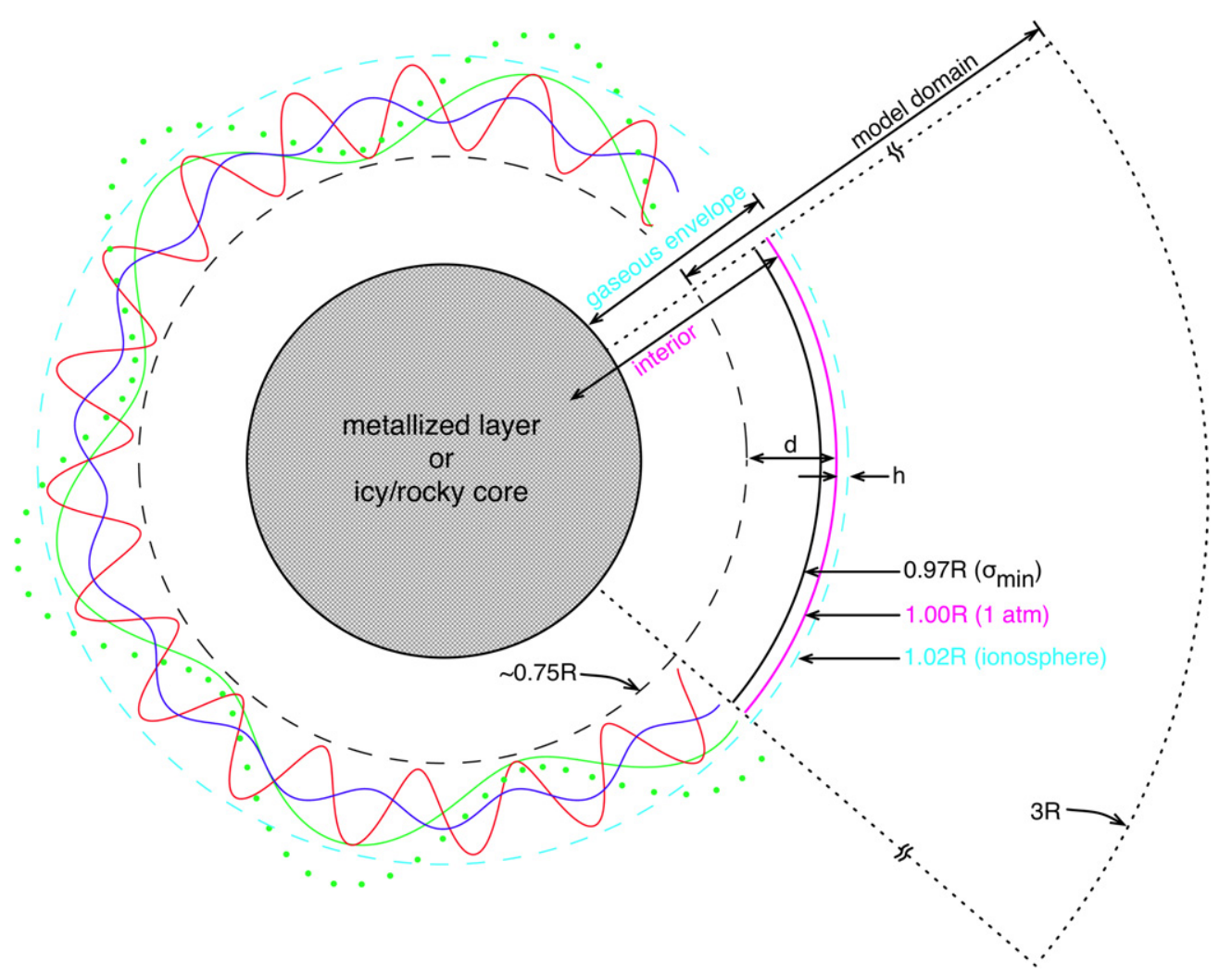

Figure 1. Sketch of the Schumann resonance phenomenon in planetary cavities. The diagram on the right-hand side illustrates the major characteristics of the cavity, including the domain of our numerical model. The radius of the planet, $R$, corresponds to an atmospheric pressure of $1 \mathrm{bar}$.

(A color version of this figure is available in the online journal.)

where $h$ is the effective height of the ionosphere, $\varepsilon_{r}$ is the relative permittivity, $\sigma$ is the conductivity of a uniform medium, and $\varepsilon_{o}$ is the permittivity of vacuum. The outer boundary is chosen such that the skin depth, $\delta_{h}$, is much smaller at its location than the effective height of the ionosphere, and

$$
\delta_{h} \cong \sqrt{\frac{2}{\mu_{o} \omega \sigma}} \ll h,
$$

with $\mu_{o}$ the permeability of vacuum and $\omega$ the angular frequency of the normal mode. Although merely valid under the assumptions $h \ll R, \sigma \ll \omega \varepsilon_{o}$, and medium uniformity, Equation (2) provides a simple method for assessing the eigenfrequency variation with cavity thickness and medium losses; increasing these two parameters decreases the eigenfrequencies.

In addition to the eigenfrequencies, the cavity is characterized by a second parameter, known as the $Q$-factor, which measures the ratio of the accumulated field power to the power lost during one oscillation period. The $Q$-factor measures the wave attenuation in the cavity and is defined by

$$
Q_{n} \equiv \frac{\operatorname{Re}\left(f_{n}\right)}{2 \operatorname{Im}\left(f_{n}\right)} \approx \frac{f_{n}^{p}}{\Delta F_{n}^{p}},
$$

where $R e$ and $I m$ are the real and imaginary parts of $f_{n}$, and $\Delta f_{n}^{p}$ is the FWHM of peak $n, f_{n}^{p}$, in the Schumann resonance frequency. Good propagation conditions in the cavity $\left(\operatorname{Im}\left(f_{n}\right) \rightarrow 0\right)$, i.e., low wave attenuation, imply narrow spectral lines and, consequently, high $Q$-factors.

Modeling ELF wave propagation on Earth is relatively straightforward compared to other planetary environments because several approximations are acceptable, namely (1) the cavity is thin, (2) the surface is a perfect electric conductor, (3) the conductivity profile is approximately exponential, and (4) atmospheric permittivity corrections can be neglected. These conditions allow for longitudinal and transverse modes decoupling and simplify the analytical calculations (Greifinger \& Greifinger 1978; Sentman 1990a). Unlike that of Earth, thick cavities containing dense atmospheres and sometimes undefined surfaces are more difficult to investigate, and numerical modeling is required. In this work, we use a finite element model previously employed to the study of TLF-ELF wave propagation in planetary cavities. This model solves Maxwell equations under full wave harmonic propagation (Simões 2007; Simões et al. 2007), eigenmode (Simões et al. 2008a, 2008b), and transient formalisms (Simões et al. 2009).

\section{SCHUMANN RESONANCES ON EARTH AND PLANETARY CONTEXT}

\subsection{Earth Results Summary}

In the last decades, significant accomplishments have been reported in Schumann resonance measurements and modeling. Indeed, continuous monitoring of ELF waves from multiple stations around the world has been used to investigate lightning-thunderstorm and tropospheric-ionospheric connections, because Schumann resonance signatures are mostly driven by lightning activity and ionosphere variability. The interaction between the solar wind and the ionosphere distorts and modulates the upper boundary and the resultant cavity eigenfrequencies. The Schumann resonance signatures therefore vary over the 11 year solar cycle, as well as shorter temporal events such as solar flares; observations also show that the resonance 
amplitude, frequency, and cavity $Q$-factor vary during solar proton events. Another major interest of Schumann resonance studies on Earth is concerned with the processes linking lightning and thunderstorm activity to the global electric circuit. Currently, Schumann resonance studies of the Earth-ionosphere cavity are driven by three major research fields related to atmospheric electricity, specifically (1) the global electric circuit and transient luminous events such as sprites, (2) tropospheric weather and climate change, and (3) space weather effects. The most important Schumann resonance characteristics measured on the ground include: $f \cong 7.8,14.3,20.8,27.3,33.8 \mathrm{~Hz}, \ldots$, $Q \sim 5, E \sim 0.3 \mathrm{mVm}^{-1} \mathrm{~Hz}^{-1 / 2}$, and $B \sim 1 \mathrm{pT}$, where $E$ and $B$ are the electric and magnetic fields, respectively. This work is focused on the outer planets, so we shall not elaborate further on Earth Schumann resonance matters, but the interested reader can find additional details in several reviews (Galejs 1972; Bliokh et al. 1980; Sentman 1995; Nickolaenko \& Hayakawa 2002; Simões et al. 2011a).

\subsection{Planetary Environments}

The existence of Schumann resonances has been conjectured for most planets and a few moons. Mercury and our Moon, where lack of any significant atmosphere prevents the formation of a surface-ionosphere cavity, are obvious exceptions. Since a detailed description of each environment is not fundamental at this stage, we shall summarize the results relevant to this work only; additional information can be found elsewhere (see Simões et al. 2008c for a review). In theory, normal modes of any cavity can be excited, provided a sufficiently strong impulsive excitation source is present to generate them. If the modes are not critically damped by high conductivity within the cavity, they would form a spectrum of distinct lines at the eigenfrequencies. One of the first questions to be answered is therefore the nature of the conductivity within the ionospheric-atmospheric cavities of the various planets of the solar system.

\subsubsection{Venus}

Three major characteristics distinguish the cavity of Venus from that of the Earth: (1) the surface is not a perfect reflector of ELF waves, (2) the cavity is more asymmetric, and (3) the atmospheric density is larger. Moreover, although new reports by Russell et al. (2010) based on Venus Express data suggest that lightning activity is prevalent on Venus, the issue still remains controversial. This is mainly due to the lack of unequivocal optical observations of flashes in the clouds and a plausible required charging mechanism that will generate strong enough electrical fields to ensure breakdown in relatively short times to match the postulated rate (e.g., Yair et al. 2008). There are nevertheless at least two works claiming observation of optical lightning on Venus: one performed on board Venera 9 (Krasnopol'sky 1980) and another with a terrestrial telescope (Hansell et al. 1995). On the other hand, Michael et al. (2009) suggest that low abundance of aerosols and high conductivity of the atmosphere appear to rule out lightning activity, at least in the $40-70 \mathrm{~km}$ altitude region. According to their study, the ratio of negative to positive charges resulting from charge transfer of ions and attachment of electrons in the clouds is found to be very large, preventing electric field effective buildup. In addition to optical and whistler dispersion signatures, investigation of Schumann resonance on Venus would provide complementary means to infer electric activity and potentially settle the disagreement. Although the expected Schumann eigenfrequencies are similar to those of Earth, surface losses can possibly lower the frequencies by as much as $\sim 1 \mathrm{~Hz}$ compared to those expected in a cavity with perfectly reflecting surface. Cavity asymmetry partially removes eigenmode degeneracy and line splitting should be more marked than on Earth $(\sim 1 \mathrm{~Hz})$. ELF wave attenuation is smaller than on Earth and, consequently, higher $Q$-factors are expected $(Q \sim 10)$. The most interesting feature, however, might concern the electric field altitude profile. Because of a significant atmospheric density, it is predicted that the Schumann resonance electric field profile should show a maximum at an altitude of $\sim 32 \mathrm{~km}$, induced by refraction phenomena (Simões et al. 2008b), instead of a monotonic profile as on Earth. At this altitude, cavity curvature is balanced by atmospheric refraction and the wave vector is horizontal; this phenomenon is also predicted when the Fermat principle or ray tracing techniques are employed for much shorter wavelengths: at $\sim 32 \mathrm{~km}$, a horizontal light beam propagates horizontally around the planet if scattering is negligible.

$$
\text { 3.2.2 Mars }
$$

The electric environment of Mars remains uncertain despite the significant amount of data provided by several orbiters and landers over recent decades. Additionally, a highly heterogeneous surface and irregular magnetic field make models more complex and unreliable. Although the Martian cavity radius suggests higher eigenfrequencies than on Earth, the significant atmospheric conductivity decreases Schumann resonance frequencies and $Q$-factors as well. The fundamental eigenfrequency probably lies in the range $8-13 \mathrm{~Hz}$; the most significant result, though, is a low $Q$-factor $(Q \sim 2)$ that implies significant wave attenuation. Although high frequency radiation from glow discharge is plausible (Farrell et al. 1999), modeling predicts large conductivity in the Martian troposphere and stratosphere (Michael et al. 2008) that results in significant wave dumping and possibly hinders development of Schumann resonance modes. Thus, it is not clear whether triboelectric phenomena, even in massive dust storms, can sustain ELF resonances in the cavity. Interestingly, Schumann resonance monitoring could contribute to the study of a sporadic ionospheric layer probably induced by meteoroids (Molina-Cuberos et al. 2006). Attempts to remotely-sense the electromagnetic signature of the postulated electrical activity on Mars have been undertaken from Mars orbit and from Earth-based instruments. Ruf et al. (2009) conducted daily $5 \mathrm{hr}$ measurements using a new instrument on the Deep Space Network radio telescope, and reported the detection of non-thermal radiation for a few hours that coincided with the occurrence of a deep dust storm on Mars. The spectrum of the non-thermal radiation showed significant peaks around predicted values of the lowest three modes of the Martian Schumann resonance (e.g., Pechony \& Price 2004). Since Schumann resonance radiation is formed by discharges exciting the surface-ionosphere cavity, Ruf et al. (2009) interpreted their observations as indicative for the occurrence of lightning within the dust storm. However, the ELF peaks reported imply large $Q$-factors $(Q>100)$ and are almost equally spaced over the frequency range, contradicting a straightforward Schumann resonance interpretation. Anderson et al. (2011) used the Allen Telescope Array in an attempt to corroborate the previous results but did not detect any non-thermal emission associated with electrostatic discharges; it is nevertheless important to emphasize that they did not detect large-scale dust storms either. Gurnett et al. (2010) used the Mars Express MARSIS instrument to look for impulsive radio signals from lightning discharges of Martian dust storms and reported negative results. 
The search covered $\sim 5$ years of data and spanned altitudes from $275 \mathrm{~km}$ to $1400 \mathrm{~km}$ and frequencies from 4.0 to $5.5 \mathrm{MHz}$, with a time resolution of $91.4 \mu \mathrm{s}$ and a detection threshold of $2.8 \times$ $10^{-18} \mathrm{~W} \mathrm{~m}^{-2} \mathrm{~Hz}^{-1}$. At comparable altitudes the intensity of terrestrial lightning is several orders of magnitude above this threshold. Although two major dust storms and many small storms occurred during the search period, no credible detections of radio signals from lightning were observed. The claim of Schumann resonance detection on Mars must be interpreted with extreme caution and requires confirmation.

\subsubsection{Titan}

Titan, the largest moon of Saturn, is the only body, other than Earth, where in situ measurements related to Schumann resonance have been attempted. Although convective clouds and storm systems have been detected in Cassini images, their composition, dynamics, and microphysics seem to be unconducive to the emergence of electrical activity (Barth \& Rafkin, 2010). And indeed, despite repeated passages near Titan, Cassini did not detect any radio signature that can be attributed to lightning (Fischer \& Gurnett 2011). The Huygens probe did record ELF spectra during the descent upon Titan that exhibit a peak close to $36 \mathrm{~Hz}$ (Fulchignoni et al. 2005; Grard et al. 2006). Several laboratory tests on the flight spare and mockup models, including antenna boom vibration at cryogenic temperatures, revealed no artifact at the same frequency. In spite of progresses in Titan cavity modeling, the nature of this signal remained unclear for a while because the electric field signature was not fully consistent with that of a Schumann resonance (Simões et al. 2007; Béghin et al. 2007). The few VLF events recorded by Huygens, if related at all to lightning activity, imply a much lower flash rate than on Earth (Hofe 2005; Simões 2007), inconsistent with the magnitude of the $36 \mathrm{~Hz}$ spectral line (Béghin et al. 2007). Presently, the most promising mechanism that could explain the Huygens measurements involves an ionacoustic turbulence resulting from the interaction of Titan with the magnetosphere of Saturn (Béghin et al. 2007, 2009). Since Titan's surface is a weak reflector $\left(\delta_{\mathrm{h}}>10^{3} \mathrm{~km}\right)$, ELF waves would propagate in the subsurface down to a depth where they would be reflected $\left(\delta_{\mathrm{h}}<10 \mathrm{~km}\right)$ by a water-ammonia liquid interface (Simões et al. 2007). Theoretical models predict the existence of a subsurface ocean (e.g., Lunine \& Stevenson 1987; Tobie et al. 2005), and the Huygens probe measurements have been used for constraining the solid-liquid interface depth (Béghin et al. 2012). From a comparative planetology perspective, the surface properties of Titan fall between those of a perfect reflector, like on Earth, and those of a fuzzy, ill-defined surface, like on the giant planets.

\subsubsection{Giant Planets}

To our knowledge, only two works on TLF-ELF wave propagation and Schumann resonance in the giant planets have previously been published. Sentman (1990b) calculated the Schumann resonance parameters for Jupiter by computing from first principles the conductivity profile of shallow interior, then by assuming a perfectly conducting ionosphere estimating the eigenfrequencies and $Q$-factors. Since Jupiter's radius is one order of magnitude larger than that of Earth, the expected Schumann resonances are about 10 fold smaller (Equation (1)). Simões et al. (2008a) considered improved conductivity profiles and also included the permittivity contribution because the cavity's inner boundary is located deep within the gaseous envelope, where refraction phenomena play a role. In the latter work, the wave propagation model was generalized to the other giant planets because similar conditions apply. Unlike the Jovian planets where measurements provided some atmospheric composition constrains, the water content uncertainty in the fluid envelopes of Uranus and Neptune is significant, implying electric conductivity profiles possibly differing by several orders of magnitude (Liu 2006; Liu et al. 2008). Simões et al. (2008a) showed that Schumann resonance measurements could be used to constrain the conductivity profile and the water content. The detection by $C / N O F S$ of ELF waves leaking into space from the Earth surface-ionosphere cavity prompts a new approach for the investigation of Schumann resonances in other planets and, consequently, of the water content in their gaseous envelopes.

\section{$3.3 \mathrm{C} / \mathrm{NOFS}$ Measurements}

The Vector Electric Field Instrument (VEFI) on the $C / N O F S$ satellite offers new capabilities for the investigation of planetary atmospheric electricity, demonstrating that ELF wave detection no longer requires in situ techniques. VEFI consists primarily of three orthogonal $20 \mathrm{~m}$ tip-to-tip double probe antennas (Pfaff 1996) and is dedicated to the investigation of ionospheric irregularities, namely spread-F and related phenomena, and to the improvement of space weather forecast. The instrument measures AC and DC electric and magnetic fields; it also includes lightning optical detectors and a Langmuir probe (Pfaff et al. 2010). In the nominal mode, the VEFI electric field sampling is $512 \mathrm{~s}^{-1}$, with sensitivity better than $10 \mathrm{nVm}^{-1} \mathrm{~Hz}^{-1 / 2}$. Remarkably, $C / N O F S$ detected Schumann resonances from orbit, in the altitude range of $400-850 \mathrm{~km}$, above the ionospheric F-peak, i.e., outside the surface-ionosphere cavity. These signatures are unambiguous, and more perceptible and clear under specific conditions: in a quiet ionosphere, during nighttime, over equatorial regions developing mesoscale convective systems, while intense lightning bursts are seen. Figure 2 shows typical electric field data recorded on 2008 May 31 during minimum solar activity. Spectrograms of the meridional/vertical and zonal/horizontal components are presented, as well as mean spectra integrated through the whole orbit for better peak visualization. Data are calibrated but intentionally not filtered to illustrate VEFI measurements robustness, namely instrument sensitivity and Schumann resonance features resolution. The Schumann resonance amplitude varies between about $0.01-0.1$ and $0.1-3 \mu \mathrm{Vm}^{-1} \mathrm{~Hz}^{-1 / 2}$ during day and nighttime, respectively. During nighttime, the average electric field is $\sim 0.25 \mu \mathrm{Vm}^{-1} \mathrm{~Hz}^{-1 / 2}$ in the altitude range covered by $C / N O F S$. Based on modeling, plasma anisotropy seems to allow ELF wave propagation through the ionosphere in the plane perpendicular to the magnetic field (e.g., Madden \& Thompson 1965; Grimalsky et al. 2005), bearing resemblance to resonance tunneling phenomena of waves in stratified cold plasma (Budden 1979). Although more elaborate modeling is necessary to understand the leakage mechanism thoroughly, propagation in the whistler and extraordinary modes seem compatible with the observed results. These $C / N O F S$ findings suggest that new remote sensing capabilities for atmospheric electricity investigations in the vicinity of planets possessing an internal magnetic field could be envisaged from an orbiter.

\section{OUTER PLANETS DYNAMICS AND EVOLUTION}

\subsection{Giant Planets Composition}

The formation of the gaseous giant planets remains a mystery because current theories are incapable of explaining how 

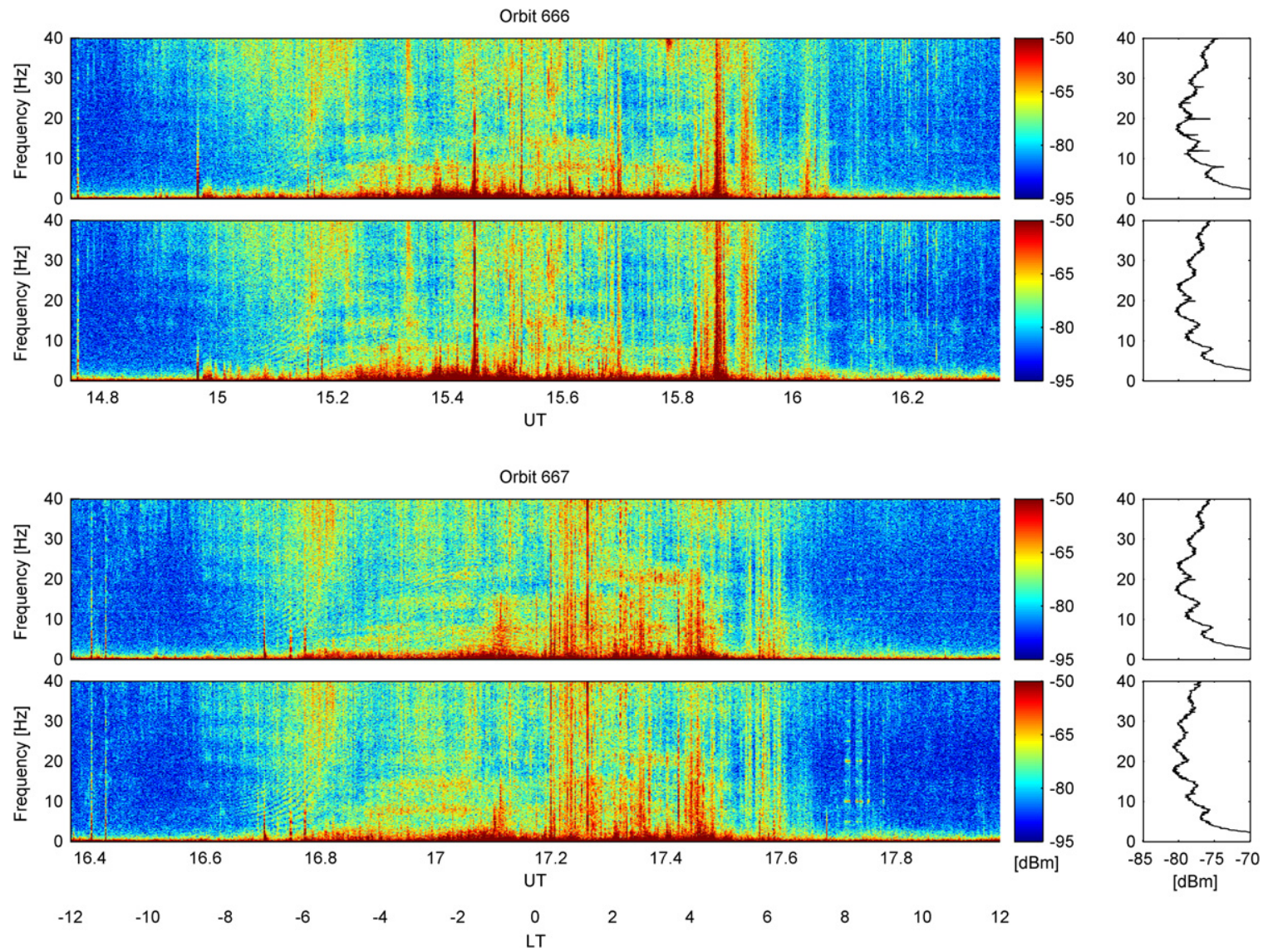

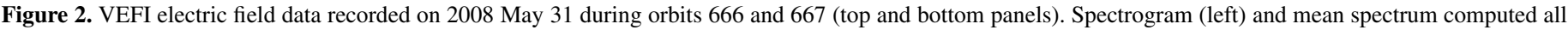

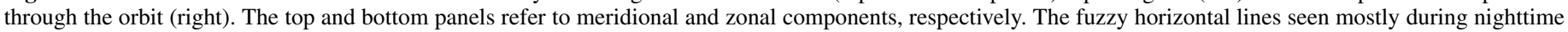
in the left panels and the spectral peaks on the right-hand side correspond to Schumann resonance eigenmodes.

their cores can form fast enough and accumulate considerable amounts of gas before the protosolar nebula disappears. In fact, the lifetime of the protoplanetary disk seems to be shorter than the time necessary for planetary core formation. Another open question related to the giant planets formation is their migration. Likely, interaction with the disk causes rapid inward migration and planets would reach the inner regions of the solar system still as sub-Jovian objects, i.e., mostly as solid bodies (e.g., Benz et al. 2000). On the other hand, according to the nebular hypothesis, Uranus and Neptune are currently located where the low density of the protoplanetary disk would have made their formation improbable. They are believed to have formed in orbits near Jupiter and Saturn and migrated outward to their present positions (e.g., Kallenbach et al. 2003). The unknown abundance of volatiles in the protosolar nebula leads to uncertainty on its gravitational and thermodynamic parameters and hampers the development of accurate accretion models (Guillot 2005). Therefore, an accurate assessment of the ice fraction of volatiles in the giant planets is required for providing a better estimate of the protoplanetary disk initial composition and an improved model of the solar system evolution.

\subsection{Jupiter and Saturn}

The atmospheres of the Jovian planets are mainly composed of hydrogen and helium with minor mole fractions of other constituents, namely ammonia, methane, and water. Although remote sensing or in situ measurements of Jupiter and Saturn atmospheres have been made, the global composition, and water content in particular, remains uncertain. Additionally, a generalization of the atmospheric composition to the entire fluid envelope may be too broad. In the present work, we consider the conductivity profiles computed by Sentman (1990b), Nellis et al. (1996), and Liu (2006). The electrical conductivity of the interiors of Jupiter and Saturn is mainly due to hydrogen; the mean composition is shown in Table 1. Figure 3 shows the conductivity profile as a function of the planet normalized radial distance.

The conductivity saturation (plateau) is due to hydrogen metallization (Nellis et al. 1996) but TLF wave reflection takes place at lower depths. We consider conductivity profiles derived by Sentman (1990b) for Jupiter and by Liu (2006) and Liu et al. (2008) for Jupiter and Saturn.

\subsection{Uranus and Neptune}

Models predict that Uranus and Neptune (called the Uranian planets in the rest of the paper) have similar internal structure (e.g., Lewis 1995). Estimations based on physical characteristics such as mass, gravity, and rotation period, and on thermodynamic properties as well, predict that the Uranian planets have an internal rocky core (iron, oxygen, magnesium, and silicon-magnesium-silicate and iron compounds), surrounded by a mixture of rock and ice (water, ammonia, methane), and an external gaseous envelope (hydrogen and helium permeated by an unknown fraction of ice). The intermediate envelope is possibly liquid because of high pressure and temperature. Considering distances normalized to the radius of the planet, the transition between the gaseous and intermediate envelopes is located at $\sim 0.8$ and 0.84 for Uranus and Neptune, respectively. 
Table 1

Planetary Cavities Characteristics

\begin{tabular}{|c|c|c|c|c|c|}
\hline Planet & $\begin{array}{c}R \\
(\mathrm{~km})\end{array}$ & $(R-d) / R(1)$ & $\begin{array}{c}B \\
(\mu \mathrm{T})\end{array}$ & $\begin{array}{c}h \\
(\mathrm{~km})\end{array}$ & Envelope Composition \\
\hline Earth & 6378 & 1 & 32 & 100 & $0.78 \mathrm{~N}_{2} ; 0.21 \mathrm{O}_{2} ; 0.01 \mathrm{Ar}$ (water vapor up to $1 \%$ ) \\
\hline Saturn & 60,268 & 0.63 & 20 & 600 & $0.94 \mathrm{H}_{2} ; 0.06 \mathrm{He}$ (ice traces) \\
\hline Uranus & 25,559 & 0.8 & 23 & $600(?)$ & $0.74 \mathrm{H}_{2} ; 0.26 \mathrm{He}$ (ice up to $10 \%$ ) \\
\hline Neptune & 24,764 & 0.84 & 14 & $400(?)$ & $0.68 \mathrm{H}_{2} ; 0.32 \mathrm{He}$ (ice up to $10 \%$ ) \\
\hline
\end{tabular}

Notes. Comparison between Earth and giant planets cavity characteristics. The radius and magnetic field refer to equatorial values; since the magnetic field of Uranus and Neptune is irregular and strongly inclined with respect to the rotation axis, values are merely indicative because, locally, they can be fivefold higher (Ness et al. 1986; Connerney et al. 1991). The third column shows where the gaseous envelope may collapse into a solid or a liquid, and gives an indication to defining the inner boundary of the cavity. The altitude, $h$, is evaluated for $\sigma \sim 10^{-3} \mathrm{Sm}^{-1}$, corresponding to skin depths of $\sim 5$ and 10-15 km for Earth and the giant planets, respectively (cf. Simões et al. 2008b). The composition of the envelope is selected from Lewis (1995) and Liu (2006) and merely indicative due to significant uncertainty. However, further explanation is useful: on Earth, when water vapor is included, composition exceeds $100 \%$-in general, composition refers to a dry atmosphere; on the giant planets, impurities contribution is unknown and composition refers to the expected envelope mean composition rather than atmospheric values.

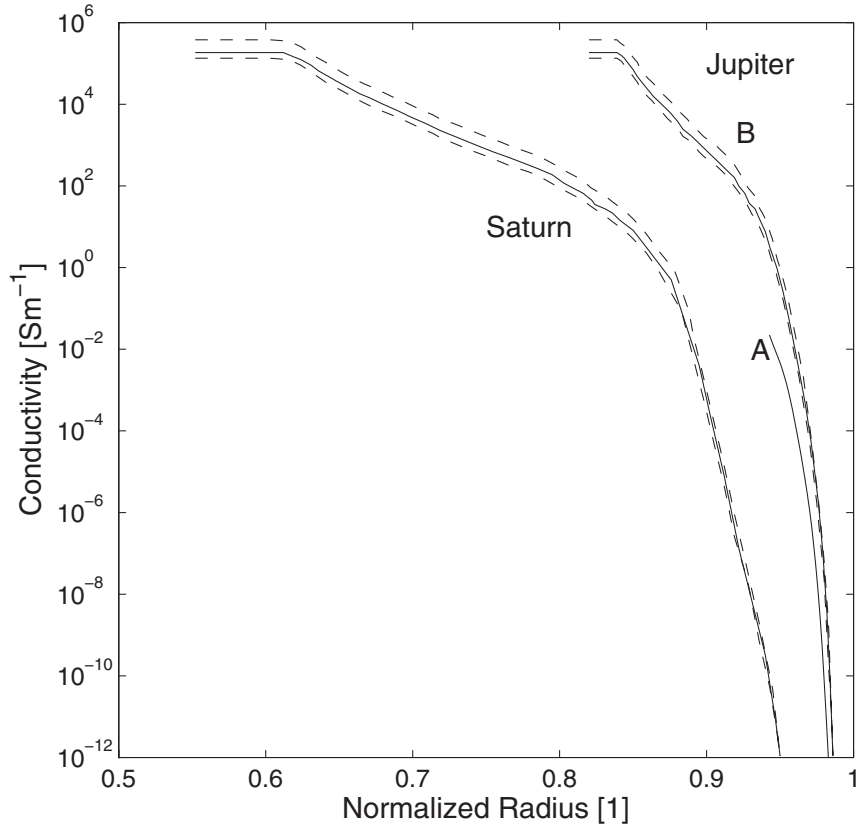

Figure 3. Conductivity profile of Jupiter and Saturn as a function of normalized radius, $\hat{R}$, where $\hat{R}=1$ corresponds to an atmospheric pressure of 1 bar. The solid and dashed lines represent the mean and uncertainty envelope of the conductivity. The profiles Jupiter-B and Saturn are adapted from Liu (2006). The profile Jupiter-A is taken from Sentman (1990b).

In the present study, we are mainly concerned with the properties of the outer layer, the gaseous envelope, where TLF-ELF waves would propagate. Figure 4 shows the conductivity profiles of the interior of Uranus and Neptune as functions of the normalized radial distance. The sharp variation in conductivity coincides with the transition between the outer and intermediate envelopes. Conductivity may vary significantly, depending on the water ice mixing ratio in the gaseous envelope. For the same depth, a water mixing ratio of 0.1 might increase the conductivity by as much as 10 orders of magnitude compared to that of a dry envelope, a fact that clearly illustrates the extreme sensitivity of TLF-ELF wave propagation conditions to the gaseous envelope water mixing ratio.

Unlike Jupiter and Saturn, the magnetic fields of the Uranian planets are quite unusual. The magnetic fields of Uranus and Neptune are tilted by $59^{\circ}$ and $47^{\circ}$ with respect to the axes of rotation, and are also displaced from the planet's center

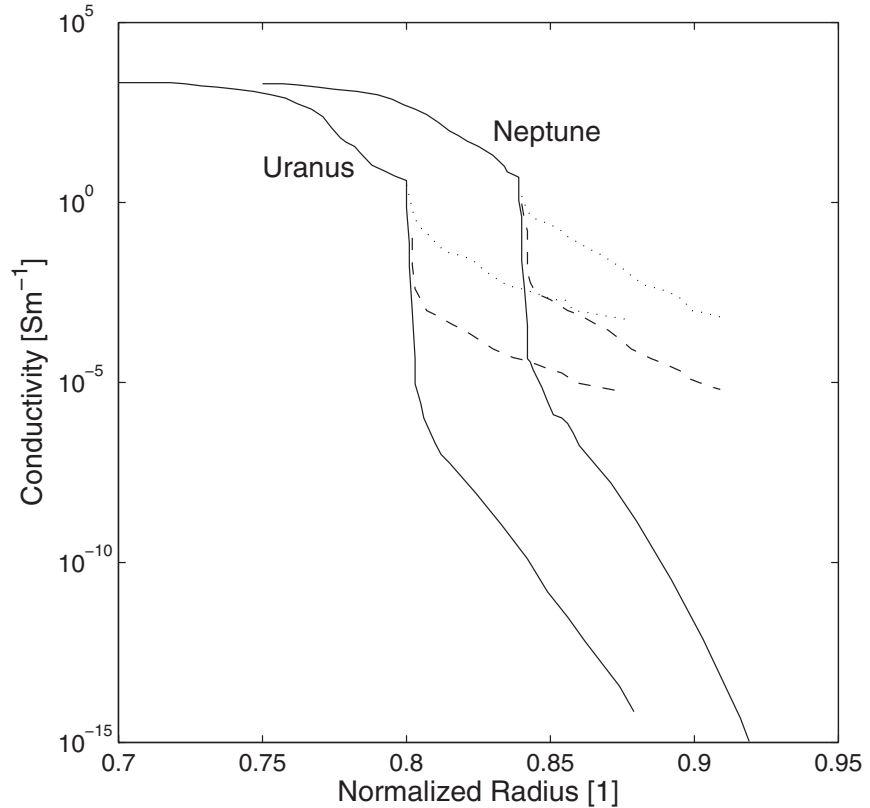

Figure 4. Conductivity profile of Uranus and Neptune as a function of normalized radius, $\hat{R}$, where $\hat{R}=1$ corresponds to an atmospheric pressure of 1 bar. The solid, dashed, and dotted lines correspond to $0,0.01$, and 0.1 water content, respectively. The conductivity profiles are adapted from Liu (2006) and Liu et al. (2008).

by $0.31 R_{\mathrm{U}}$ and $0.55 R_{\mathrm{N}}$, respectively. This atypical magnetic field structure results in highly asymmetric magnetospheres and suggests that it is generated in the intermediate, possibly liquid, envelope rather than in the core itself as in the other planets (Ness et al. 1986; Connerney et al. 1991). In addition to a strong quadrupolar moment contribution, Uranus sideways rotation complicates even further the magnetic field distribution. The magnetic field distribution is a second order correction for eigenfrequency and $Q$-factor assessments because the medium is highly collisional in most of the envelope. However, the magnetic field correction is fundamental to investigate the cavity leakage. The equatorial magnetic fields are given in Table 1.

\section{NUMERICAL MODEL}

The cavities of the gaseous giant planets are intricate, and so the standard analytical approximations used for Earth are unsuitable; thus, numerical modeling is necessary. We use an 
approach similar to that employed by Simões et al. (2008a, 2008 b) to study TLF-ELF wave propagation in planetary environments. The numerical model is based on the finite element method and solves Maxwell equations with specific boundary conditions and medium properties. The algorithm calculates the eigenfrequencies, $Q$-factors, and electromagnetic field distribution within the cavity. The most important parameters for running the numerical model include: (1) the conductivity profile of the atmosphere and ionosphere, $\left(\sigma_{\text {iono }}\right),(2)$ the conductivity profile of the interior $\left(\sigma_{\text {int }}\right),(3)$ the permittivity profile of the interior $\left(\varepsilon_{\text {int }}\right),(4)$ the depth of the inner boundary $(d)$, and $(5)$ the height of the outer boundary $(h)$. The inner and outer boundaries are located where $\delta_{h} \ll d$ and $\delta_{h} \ll h$, respectively, where $\delta_{h} \sim$ $10 \mathrm{~km}$. The inner boundary coincides roughly with the interface between the gaseous envelope and the metal (Jupiter, Saturn) or icy/liquid (Uranus, Neptune) medium (Liu 2006; Liu et al. 2008).

The atmospheric conductivity is computed from the electron density and collision frequency profiles, which are derived from pressure, temperature, and composition data recorded during several missions, namely Pioneer, Voyager, Galileo, and Cassini. The conductivity profiles of the planetary interiors are shown in Figures 3 and 4. Since density increases with depth and the vacuum approximation is no longer valid, we employ the approach of Simões et al. (2008a, 2008b) to derive the permittivity of the interior of the giant planets, assuming that: (1) the refractivity is a linear function of gas density, (2) the medium response can be extrapolated from the radiofrequency to the TLF-ELF range, i.e., non-dispersive medium conditions at low frequency apply, (3) the contributions other than that of hydrogen are neglected (more elaborate approaches are considered if the water content ratio exceeds $\sim 0.1 \%$ ), and (4) the relative permittivity of liquid hydrogen is $\sim 1.25$. A more elaborated analysis of refractivity effects in ELF wave propagation can be found elsewhere (Simões et al. 2008b). We first employ the eigenvalue analysis to determine the eigenfrequencies and $Q$-factors of isotropic cavities (Simões et al. 2008a). For a qualitative estimation of the cavity leakage, the electric and magnetic fields are computed with a full wave harmonic propagation algorithm in an anisotropic medium. For the sake of simplicity, we employ a vertical Hertz dipole to model the electromagnetic sources (Simões et al. 2009) and consider a dipolar static magnetic field of known magnitude at the equator (Table 1).

In addition to the conductivity profile variability with water content, estimates of the TLF-ELF wave magnitude resulting from cavity leakage are invaluable for establishing the detection range and defining instrumentation requirements. We therefore use a full wave harmonic propagation model to compute the electric and magnetic field amplitudes as function of distance to the source. The open boundary $(r \rightarrow \infty)$ is approximated by a Perfectly Matched Layer (PML) placed at $r \sim 10^{2} R$. The PML approach is used to avoid wave reflection on the edge of the domain. We consider a vertical Hertz dipole radiating in the TLF range, of arbitrary amplitude and located at $r=R$, and compute the electromagnetic field distribution inside and outside the cavity. A similar approach to that applied by Simões et al. (2009) to the Earth cavity in the VLF range is employed here to derive the conductivity tensor on the giant planets, i.e., taking into account the Pedersen and Hall conductivity corrections. The conductivity tensor is derived from the Appleton-Hartree dispersion relation that describes the refractive index for electromagnetic wave propagation in cold magnetized plasma.
The present numerical model has already been used for estimating eigenfrequencies of planetary environments and has been validated against Earth cavity data, namely ELF spectra and atmospheric conductivity. Consistent results are therefore expected as long as the conductivity profiles are reliable. For the sake of simplicity, we consider a scalar conductivity to evaluate cavity eigenvalues because anisotropic corrections (Budden 1979; Simões et al. 2009) are small compared to the conductivity profile uncertainty. Nonetheless, the conductivity tensor is included in the full wave harmonic propagation model to compute the electric and magnetic field amplitude resulting from cavity leakage, which allows for spacecraft-planet distance versus instrument sensitivity assessments. We choose 2D axisymmetric approximations whenever possible and 3D formulations otherwise.

\section{RESULTS}

In this work we address wave propagation primarily in the Uranian planets for the following reasons. First, the major objective is to investigate the suitability of the proposed technique for estimating the water content in the gaseous envelopes from Schumann resonance measurements. Second, water content uncertainty in the gaseous envelope of Uranus and Neptune is large, and therefore the technique proposed here would be more valuable for those environments. Third, unless significantly different conductivity profiles are conjectured, the eigenfrequencies and $Q$-factors of the cavities of Jupiter and Saturn would be similar to those reported previously (cf. Table 2 and results reported by Simões et al. 2008a). Finally, enhanced parameterizations are deemed necessary to quantify electromagnetic field leakage through planetary ionospheres, namely regarding source characteristics such as spatial and temporal variability of lightning; since the magnetic fields of the Jovian planets are stronger than those of Uranus and Neptune, anisotropic corrections should be more important there. A more elaborate model is nevertheless under development to compute wave propagation through the ionosphere, estimate cavity leakage as a function of lightning, ionospheric, and magnetospheric characteristics that may provide useful predictions for the Juno (en route to Jupiter) and Cassini (currently operating in orbit at Saturn) missions or future endeavors.

Voyager 2 measured the ionospheric electron density profile (Lindal et al. 1987; Tyler et al. 1989; Lindal 1992) with some discrepancy between ingress and egress, especially in the case of Uranus. Two conductivity profiles of the atmosphere and ionosphere are derived for Uranus from the Voyager data sets, based on analogies with Earth aeronomy and modeling; in the case of Neptune, a single profile is used (Capone et al. 1977; Chandler \& Waite 1986). Since the eigenfrequencies are affected little by atmospheric conductivity uncertainties due to the dominance of the interior contribution, the present model takes into account deeper variability only. Supplementary information regarding the calculation of atmospheric and ionospheric conductivity profiles of the giant planets can be found elsewhere (Sentman 1990b; Simões et al. 2008a).

In the case of the Jovian planets, where the water content uncertainty appears to be smaller than for Uranus and Neptune, we consider the conductivities shown in Figure 3 and compute eigenfrequencies and $Q$-factors of the mean, maximum, and minimum profiles. Table 2 shows the results of the eigenfrequencies and $Q$-factors of the three lowest eigenmodes of Jupiter and Saturn. Although the conductivity profile uncertainty produces minor variations in eigenfrequency and $Q$-factors, Schumann 
Table 2

Planetary Cavities Computed Eigenfrequencies

\begin{tabular}{|c|c|c|c|c|c|c|c|c|c|c|}
\hline \multirow[t]{3}{*}{ Conductivity Profile } & \multirow[t]{3}{*}{ Mode } & \multicolumn{8}{|c|}{ Planet } & \multirow[t]{3}{*}{ Reference } \\
\hline & & \multicolumn{2}{|c|}{ Jupiter } & \multicolumn{2}{|c|}{ Saturn } & \multicolumn{2}{|c|}{ Uranus } & \multicolumn{2}{|c|}{ Neptune } & \\
\hline & & $f(\mathrm{~Hz})$ & $Q[1]$ & $f(\mathrm{~Hz})$ & $Q[1]$ & $f(\mathrm{~Hz})$ & $Q[1]$ & $f(\mathrm{~Hz})$ & $Q[1]$ & \\
\hline \multirow[t]{3}{*}{ Minimum } & 1 & 0.736 & 7.022 & 0.772 & 3.672 & 2.429 & 19.01 & 2.521 & 15.46 & \multirow[t]{9}{*}{ Liu (2006) } \\
\hline & 2 & 1.308 & 6.816 & 1.401 & 3.705 & 4.245 & 17.54 & 4.416 & 15.52 & \\
\hline & 3 & 1.885 & 6.844 & 2.039 & 3.707 & 6.036 & 16.34 & 6.293 & 15.66 & \\
\hline \multirow[t]{3}{*}{ Intermediate } & 1 & 0.734 & 7.896 & 0.763 & 3.597 & 1.025 & 4.08 & 1.109 & 2.04 & \\
\hline & 2 & 1.296 & 7.913 & 1.386 & 3.252 & 1.992 & 4.06 & 2.030 & 2.11 & \\
\hline & 3 & 1.855 & 7.717 & 2.030 & 3.037 & 3.037 & 4.93 & 2.961 & 1.75 & \\
\hline \multirow[t]{3}{*}{ Maximum } & 1 & 0.752 & 9.791 & 0.767 & 4.062 & $\times$ & $\times$ & $\times$ & $\times$ & \\
\hline & 2 & 1.318 & 10.85 & 1.381 & 4.275 & $\times$ & $\times$ & $\times$ & $\times$ & \\
\hline & 3 & 1.878 & 10.82 & 1.997 & 4.278 & $\times$ & $\times$ & $x$ & $\times$ & \\
\hline \multirow[t]{2}{*}{ Equatorial radius $71,500 \mathrm{~km}$} & 1 & 0.575 & 5.202 & $\ldots$ & $\ldots$ & $\ldots$ & $\ldots$ & $\ldots$ & $\ldots$ & \multirow[t]{7}{*}{ Sentman (1990b) } \\
\hline & 2 & 1.017 & 5.938 & $\ldots$ & $\ldots$ & $\ldots$ & $\ldots$ & $\ldots$ & $\ldots$ & \\
\hline \multirow{2}{*}{ Mean radius $69,900 \mathrm{~km}$} & 2 & 1.040 & 5.625 & $\ldots$ & $\cdots$ & $\cdots$ & $\cdots$ & $\cdots$ & $\cdots$ & \\
\hline & 3 & 1.495 & 6.218 & $\ldots$ & $\ldots$ & $\ldots$ & $\ldots$ & $\ldots$ & $\ldots$ & \\
\hline \multirow[t]{3}{*}{ Polar 66,850 km } & 1 & 0.616 & 5.047 & $\ldots$ & $\ldots$ & $\ldots$ & $\ldots$ & $\ldots$ & $\ldots$ & \\
\hline & 2 & 1.100 & 5.234 & $\ldots$ & $\ldots$ & $\ldots$ & $\ldots$ & $\ldots$ & $\ldots$ & \\
\hline & 3 & 1.588 & 5.318 & $\cdots$ & $\ldots$ & $\ldots$ & $\ldots$ & $\ldots$ & $\ldots$ & \\
\hline
\end{tabular}

Notes. Lowest eigenfrequencies of the giant planets for the conductivity profiles shown in Figures 3 and 4 . The maximum conductivity profiles of Uranus and Neptune, corresponding to a water mixing ratio 0.1, prevent formation of resonant modes.

resonances could be used to confirm whether the hydrogen ionization processes are realistic as function of depth, and to assess impurity mixing ratios in the envelope as well. For Jupiter, the results for conductivity profiles derived by Sentman (1990b) and Liu (2006) produce somewhat dissimilar eigenfrequencies and $Q$-factors due to differences in the conductivity profile. These results are also important to confirm that eigenfrequencies and $Q$-factors are more sensitive to the conductivity profile than to cavity shape, e.g., equatorial versus polar radius.

Figure 4 takes into account the water content uncertainty in the gaseous envelope of the Uranian planets and shows the consequences for the conductivity profile. Because of the significant conductivity profile uncertainty, we compute the eigenfrequencies and $Q$-factors for various cavity parameterizations. To facilitate the comparisons among various parameters, namely water mixing ratio, an exponential conductivity profile with two parameters is considered:

$$
\sigma_{\text {int }}(r)=\sigma\left(r_{o}\right) e^{\left(r_{o}-r\right) / H_{d}}=\sigma_{o} e^{\left(r_{o}-r\right) / H_{d}},
$$

where $r$ is the radial distance, $r_{o}<r<R+h, r_{o}=R-d$, and $H_{d}$ is the interior conductivity profile scale height. A conductivity profile is therefore defined by the ordered pair $\left\{H_{d}, \sigma_{\mathrm{o}}\right\}$. Figures 5 and 6 show the eigenfrequencies and $Q$-factors of the cavities of Uranus and Neptune as a function of conductivity profile parameterization. Table 2 shows the three lowest eigenmodes computed for the conductivity profiles shown in Figure 4 (water content: 0, 0.01, and 0.1).

The plots presented in Figures 5 and 6 for Uranus and Neptune, respectively, correspond to specific cavity configurations, using Equation (5) and the conductivity constraints shown in Figure 4. The water content (magenta lines) is derived from the evaluations of Nellis et al. (1996) and Liu (2006). These estimates are indicative only and the results should be interpreted with caution. The conductivity profiles may be unrealistic, but they are nonetheless representative and lend themselves to a qualitative discussion that illustrates how, conversely, the Schumann resonance characterizes the conductivity profile. Figure 5 presents the eigenfrequencies and $Q$-factors of the Uranus cavity for the three lowest eigenmodes as functions of interior scale height, $H_{d}$, and interface conductivity, $\sigma_{\mathrm{o}}$. Although one eigenmode is usually sufficient to identify the corresponding exponential conductivity profile $\left(\left\{f_{1}, Q_{1}\right\} \rightarrow\left\{H_{d}, \sigma_{\mathrm{o}}\right\}\right)$, we present the eigenfrequencies and $Q$-factors of a few eigenmodes for information. The left-hand side plots in Figure 5 illustrate the importance of characterizing multiple modes with both the eigenfrequencies and $Q$-factors. These plots show two yellowish stripes corresponding to similar frequencies but different $Q$-factors; the effect is more evident in the lowest eigenmode. This effect illustrates how multiple peaks in the Schumann resonance spectrum can be used to further constrain the water mixing ratio. While the convex boundaries in Figures 5 and 6 represent the dry envelope limit, the concave ones result from multiple constraints, namely minimum conductivity close to the inner boundary, dry envelope conditions, and monotonic conductivity profiles. If the conductivity profile scale heights of Figure 4 are realistic, then the most plausible cavity parameterizations are found along the gray line. Bites in the plots top right edge are due to the lack of eigenvalues; wave resonance is hindered because a critical damping is reached caused by high water content. As expected, a combination of high $H_{d}$ and $\sigma_{\mathrm{o}}$ entails significant cavity losses and, comparatively, wave propagation conditions seem more favorable in Uranus than in Neptune, confirming previous simulations (Simões et al. 2008a). The water content affects both the frequency and $Q$-factor though more importantly in the latter (see the magenta isolines in Figures 5 and 6).

A single scale height is considered for the Uranus and Neptune interiors for the sake of simplicity, but a realistic conductivity profile is certainly more intricate. On Earth, for example, the atmospheric conductivity profile is better described by two scale heights (Greifinger \& Greifinger 1978; Sentman 1990a). An improved model that addresses a weakness in the two-scale height model is when the local scale height changes 

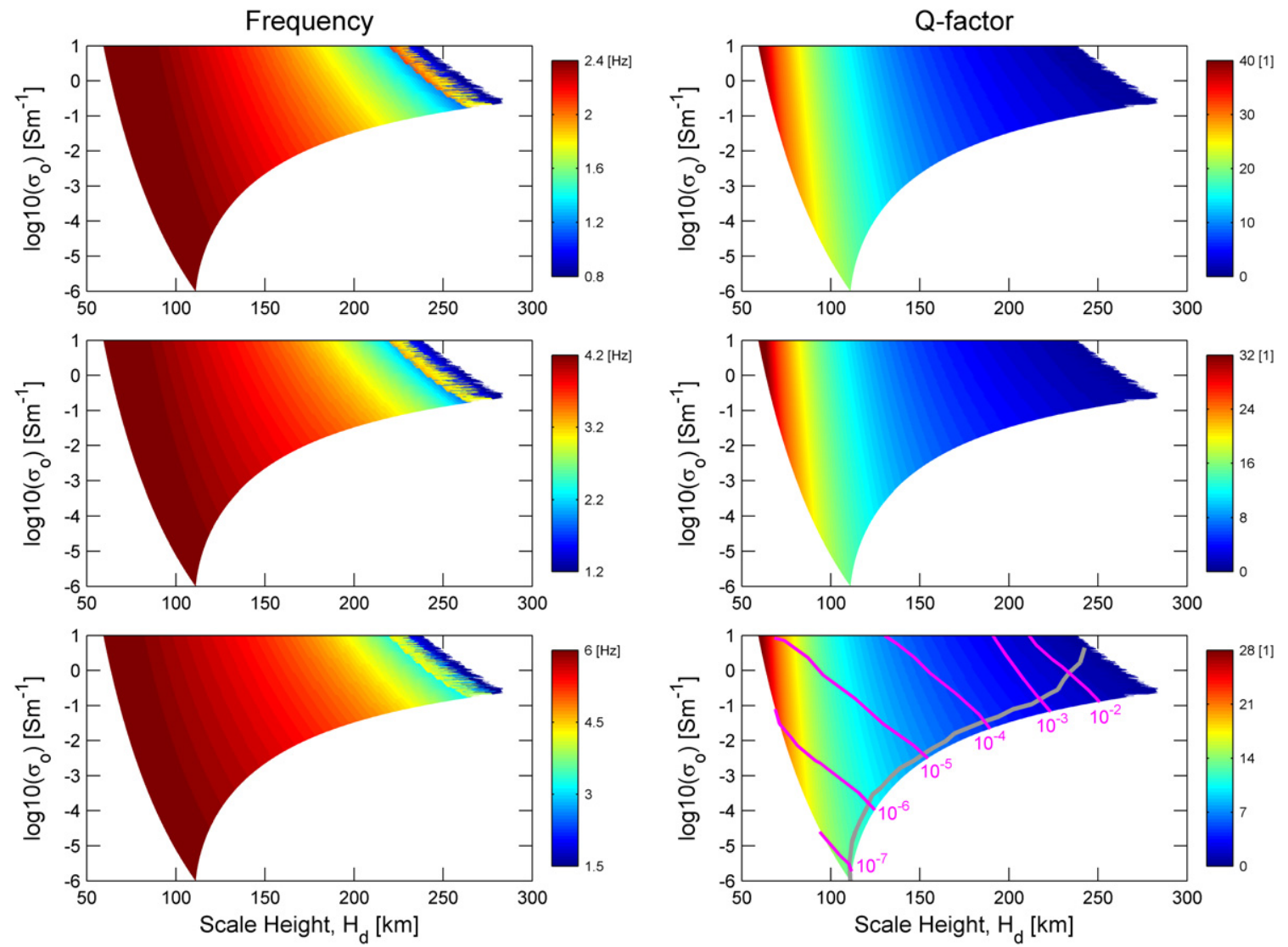

Figure 5. Modeling results of (left) eigenfrequencies and (right) $Q$-factors of (from top) the three lowest eigenmodes as function of interface conductivity ( $\sigma_{\mathrm{o}}$ ) and scale height $\left(H_{d}\right)$ of the Uranus cavity. In the bottom right panel, the magenta curves represent mean water contents in the gaseous envelope; cavity parameterizations near the gray curve represent the most plausible conductivity profiles.
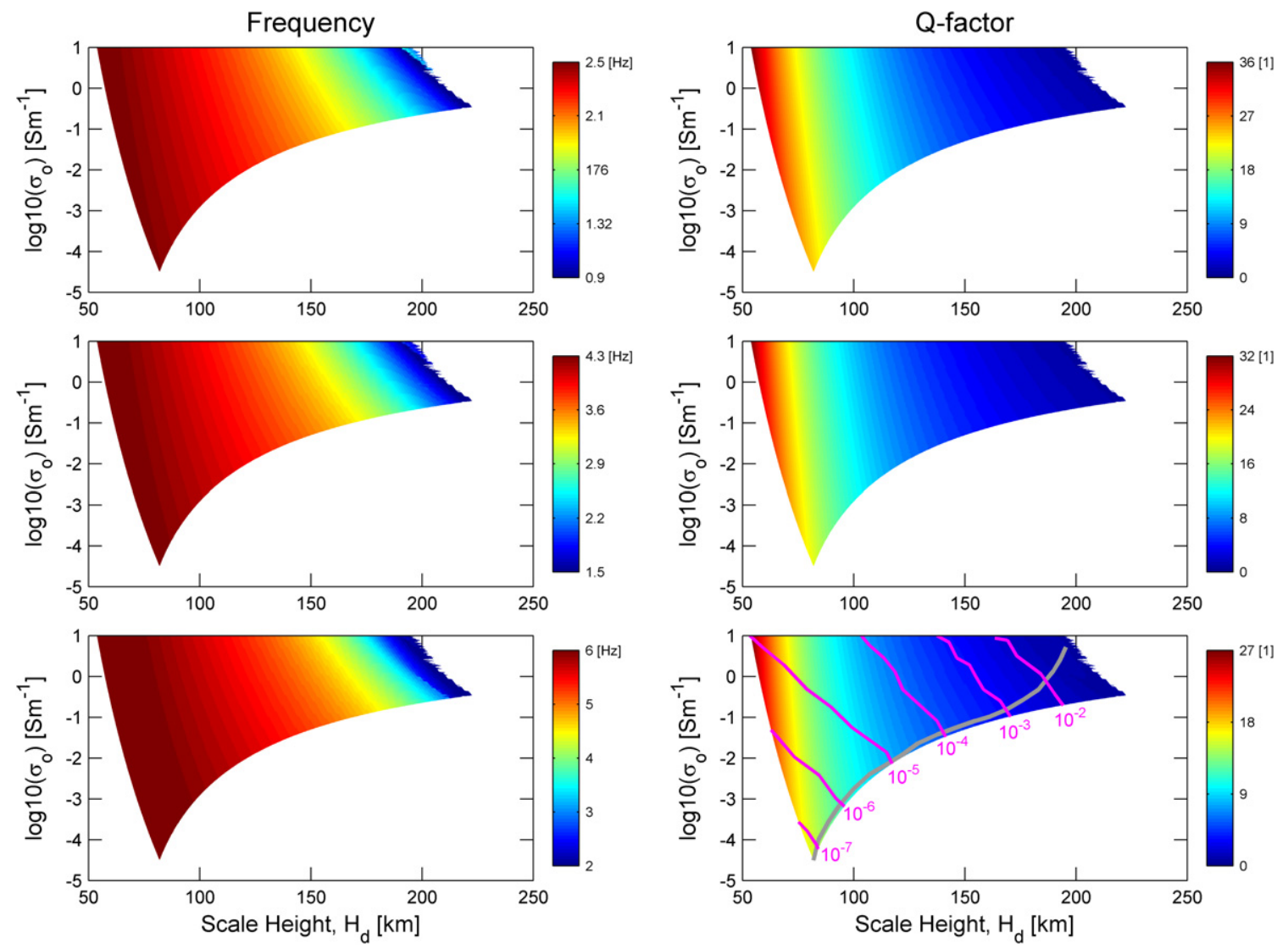

Figure 6. Same caption as in Figure 5 but for the cavity of Neptune. 


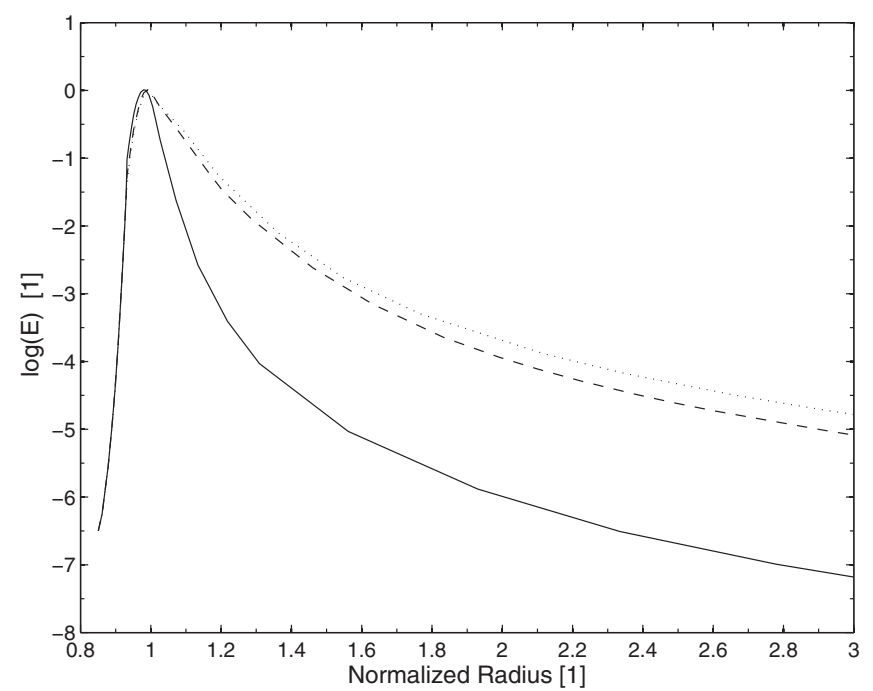

Figure 7. Normalized electric field amplitude of the lowest Schumann eigenmode as function of normalized radius of the planet, $\hat{R}$, for (dash) Uranus, (dot) Neptune, and (solid) Earth ionosphere equivalent configuration. The reference $\hat{R}=1$ corresponds to an atmospheric pressure of 1 bar.

rapidly in the region of maximum Joule dissipation, and is referred to as the "knee model," has been described by Mushtak and Williams (2002). In the case of the giant planets, mainly Uranus and Neptune, a multiple scale height profile would be preferable in order to differentiate interior, interior-atmospheric, and ionospheric parameterizations. Information from additional eigenmodes can be used to characterize conductivity profiles with multiple scale heights. In principle, the number of scale heights that can be constrained in the profile is equal to the number of spectral peaks detected, provided eigenfrequencies and $Q$-factors can be both measured accurately. The most straightforward approach would consist in solving the direct problem iteratively, starting from an initial guess and employing minimization techniques to obtain the conductivity profile best fit, which would then yield an estimation of the water content of the gaseous envelope. If possible, it should also be attempted to derive conductivity profiles directly from eigenmode information, i.e., ordered pairs $\left\{f_{\mathrm{n}}, Q_{\mathrm{n}}\right\}$.

Figure 7 shows the electric field magnitude of a radiating dipole as a function of the normalized radius of the planet. Far from the planet, where the signal resulting from cavity leakage propagates almost in a vacuum, the electromagnetic field variation with distance approaches the power law $\left(E, B \propto \mathrm{r}^{-1}\right)$ resulting from spherical wave propagating in a lossless medium. Amplitude asymptotic convergence to a theoretical solution therefore corroborates the PML approach at large distance. Since absolute comparisons are not viable because lightning stroke characteristics are unknown, a source of arbitrary amplitude is selected. In addition to the previous Uranian environments, we now consider a cavity with an ionospheric parameterization equivalent to that of Earth. Although physically not representative, this comparison determines whether detecting leakage from Uranus and Neptune cavities is more demanding than at Earth. The electric field profiles of Figure 7 suggest that wave leaking detection is more favorable than at Earth because ionospheric attenuation is weaker. Since the electron density peak in Uranus and Neptune is about two and three orders of magnitude lower than at Earth, respectively (Lindal et al. 1987; Tyler et al. 1989), integrated Pedersen and
Hall conductivities provide less wave attenuation through the ionosphere. For example, at a distance of $1.1 R$, the expected electric field would be two orders of magnitude higher than on Earth for a similar electromagnetic source. However, interpretation must be made cautiously because cavity leakage is also a function of $Q$-factor, aeronomy processes, and lightning stroke power and rate characteristics. Consequently, subsequent investigations of atmospheric electricity, namely lightning processes, are needed so that cavity leakage assessments could be improved. In theory, considering ionospheric plasma density, magnetic field parameterization and assuming similar electromagnetic source characteristics, anticipated cavity leakage in the Jovian planets is stronger than on Earth but weaker than on Uranus and Neptune.

\section{DISCUSSION}

The most accurate way of evaluating the water content profile of the giant planets is employing in situ techniques for measuring the water mixing ratio in the gaseous envelope. This approach was used by the Galileo probe Mass Spectrometer during the descent through the atmosphere of Jupiter down to $\sim 20$ bar (Mahaffy et al. 2000; Atreya et al. 2003). However, only a small fraction of the envelope has been explored. Other solutions involve Earth-orbiting observatories or dedicated spacecraft around the planets, e.g., Cassini at Saturn, employing infrared, optical, or ultraviolet spectrometry to infer atmospheric composition (e.g., Fouchet et al. 2005; Baines et al. 2009). The microwave radiometer part of Juno, a forthcoming mission to Jupiter, may provide accurate water content estimates possibly down to about 200 bar (Matousek 2007). These options are reliable and accurate but allow for estimates of the envelope outer shallow layer only, i.e., the atmosphere and ionosphere. Since the connection between water content and electric conductivity is well established, in situ measurements of the conductivity profile would provide an indirect method for water content assessments. During the descent in the atmosphere of Titan, the Permittivity, Wave, and Altimetry analyzer on board the Huygens probe performed electric conductivity measurements from about $140 \mathrm{~km}$ down to the surface (Fulchignoni et al. 2005; Grard et al. 2006; Hamelin et al. 2007; López-Moreno et al. 2008). This option would be applicable in the giant planets down to moderate depths only. Given that a connection among the planetary Schumann resonance frequencies, conductivity profile, and water content exists, TLF-ELF measurements provide a practical method for inferring the water content in the envelope. On the other hand, $C / N O F S$ data show that measurements inside the cavity are not mandatory and that a remote sensing method is likely to be practical for planets that possess a magnetic field. Additionally, unlike other solutions that offer local measurements only, Schumann resonance measurements would provide a global distribution of the conductivity profile and, consequently, better estimates of the mean water content. As shown in Table 2 and Figures 5 and 6, Schumann resonance modes can be used to estimate global water contents up to a few percent in the Uranus and Neptune gaseous envelopes and, to a lesser extent, to confirm whether the conductivity models of Jupiter and Saturn are realistic. Detection of terrestrial Schumann resonance signatures on board $C / N O F S$ unveils new remote sensing capabilities for investigating atmospheric electricity and tropospheric-ionospheric coupling mechanisms, not only on Earth but also other planetary environments that possess a magnetic field. Observation of Schumann resonances above the ionospheric F-peak was unexpected and requires revisiting 
analytical and numerical models, which are not fully consistent with $C / N O F S$ observations. However, although analytical and numerical modeling requires significant improvements, it is clear that medium anisotropy plays a key role in cavity leakage.

The snow line is an important concept to address the water ice condensation front in protoplanetary disk accretion models, to investigate convective and radiation phenomena as well as and chemical processes, and was allegedly located near the orbit of Jupiter when planets formed. The condensation front would be expanding during the solar nebula coalescence and subsequent disk accretion processes, and then receding again throughout the cooling phase. For example, Stevenson \& Lunine (1988) argue that the Galilean satellites formed later than the proto-Jupiter, allowing for late accretion of water into these moons. Estimates of the relative abundance and variability of the various elements in the solar system, in particular with respect to solar average composition, are frequently achieved from isotopic measurements. Information on the relative enrichment and depletion of the various elements is then used to investigate the early stages of the solar system. Measurements made by the Galileo probe (Mahaffy et al. 2000) in Jupiter's atmosphere found less water than expected. Several explanations have been proposed, including (1) non-representative measurements due to sampling of a dry area of the atmosphere, (2) a larger fraction of oxygen trapped in the core in the form of silicates, (3) the water ratio being lower than expected in the solar system, and (4) the snow line being located farther from the Sun, suggesting more water is diffused toward the periphery of the solar system. Relocating the snow line farther away would imply that the Uranian planets are water-enriched; in the case of Neptune, the water enrichment could be several hundred times larger (e.g., Lodders \& Fegley 1994). However, there are also theoretical models that may be consistent with water and oxygen depletion (Fegley \& Prinn 1988). Measurement of water mixing ratios in the giant planets would thus provide useful data for constraining protoplanetary disk accretion models, offering a better distribution of water throughout the solar system.

Figure 8 illustrates the rationale linking the water mixing ratio, electrical conductivity profile, remote sensing and in situ measurement techniques, Schumann resonance spectra, and protoplanetary disk parameters. The water mixing ratio in the gaseous envelope plays a key role in atmospheric chemistry, which drives the electrical conductivity profile through molecular reaction rates-e.g., recombination-and electron and ion mobility. The presence of electrophilic species, in particular, modifies the electrical conductivity by changing recombination rates and cluster ion mobility. Along with geometry parameters such as size, the conductivity profile drives the Schumann resonance spectrum in the cavity. Both TLF-ELF electric and magnetic field measurements can be used to estimate Schumann resonance signatures. Remote sensing is often more versatile than in situ measurements. For example, electric field measurements are frequently noisier on board descent probes due to shot noise, mainly below $10 \mathrm{~Hz}$. A descent vessel is also more susceptible to vibrations, which introduce additional artifacts to the spectrograms. As suggested by our calculations, high water mixing ratios would shift Schumann resonance toward lower frequencies and produce broader peaks as well as weaker signatures. In the case of Uranus and Neptune, a water mixing ratio of $\sim 0.1$ might change the frequencies and $Q$-factors by a factor of 2 and 15 compared to those related to dry envelopes. For the sake of comparison, variability of eigenfrequencies and $Q$-factors on Earth due to lightning and ionospheric dynamics is
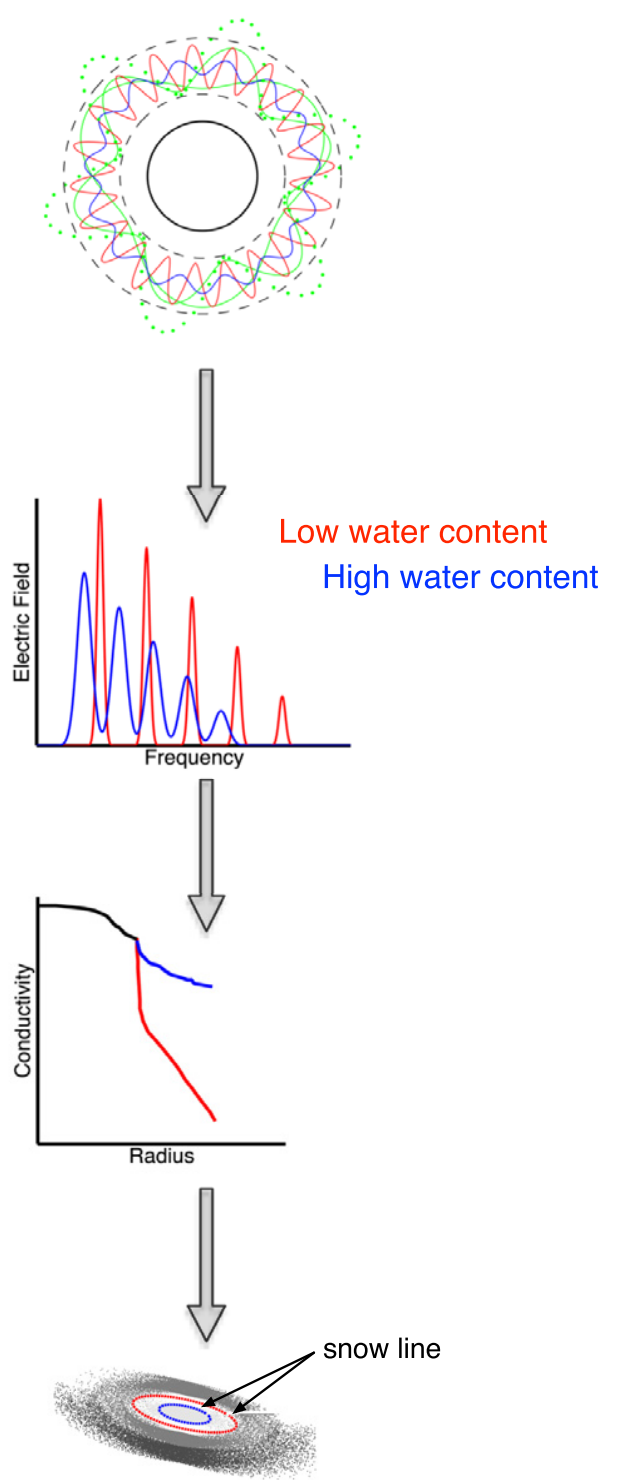

Figure 8. Illustration of the connection between the water mixing ratio, conductivity profile, Schumann resonance spectrum, cavity leakage, and water ice condensation front of the solar system. In situ and remote sensing of TLF-ELF electric and magnetic fields in the giant planets yield the Schumann resonance spectra and the respective electrical conductivity profile, which can be used to infer the average water content in the gaseous envelope and to constrain the water ice condensation front (snow line) of the protoplanetary disk from which the solar system evolved.

(A color version of this figure is available in the online journal.)

less than $10 \%$ and $50 \%$, respectively. Since a $50 \%$ enrichment or depletion of the water mixing ratio in the gaseous envelope of Jupiter with respect to the solar average has significant implications for protoplanetary disk models, discrimination between a water mixing ratio of 0.1 and 0.01 in the Uranian planets would provide key information for a better understanding of the formation and evolution of the solar system.

\section{CONCLUSION}

Limited data of volatiles abundance, namely water, ammonia, and methane in the outer planets prevent the development of accurate models of the protoplanetary disk dynamics, from which the solar system evolved. Thus, knowledge of the water 
mixing ratio in the gas giants is crucial to constraining the protosolar nebula composition. Water content estimates have been measured so far with both in situ and remote sensing techniques. These approaches generally yield local atmospheric composition only, down though to pressure levels of tens of bars. However, extrapolating local composition measurements to the whole gaseous envelope might be inappropriate, particularly at large depths.

We propose here a new approach for estimating the global water content of the giant planet envelopes from Schumann resonance measurements. Water has a clear impact on the electrical conductivity and Schumann resonance signatures. Compared to a dry gaseous envelope, the predicted eigenfrequencies of the cavity of Uranus and Neptune show a threefold decrease when the water content reaches $10 \%$. The $Q$-factors are even more sensitive and decrease by as much as a factor of 40 . We therefore advocate performing in situ and remote sensing TLF-ELF electric and magnetic field measurements to probe the water global distribution in the gaseous envelopes, at depths of hundreds, possibly thousands, of kilometers. As seen from the $C / N O F S$ satellite ELF spectra, Schumann resonance detection from orbit is feasible, which presents an obvious advantageous compared to in situ observations. Assuming similar lightning characteristics, preliminary models shows that wave leakage in the outer planets would be stronger than on Earth, suggesting detection of Schumann resonance signatures may even be easier there. Identification of multiple peaks from TLF-ELF spectra would further improve the conductivity profile and corresponding water content estimates. Combining both remote sensing and in situ techniques would of course strengthen synergistic analyses of the volatiles composition.

A Schumann resonance spectrum will be excited in the cavity of the gaseous giants if there are sufficiently powerful electrical drivers, such as lightning. Modeling confirms that with plausible conductivity profiles the distinctive resonance spectrum will form and therefore be usable for probing the conductivity of the shallow interior of the planets. Electric and magnetic antennas could therefore be used not only to study atmospheric electricity and wave propagation but to estimate water content in the gaseous envelopes, to infer the volatile abundance in the protosolar nebula from which the solar system evolved, and to constrain the water ice condensation front and better locate the snow line in protoplanetary disk accretion models. The accurate assessment of the water content in the giant planets could also perhaps contribute to the understanding of the formation and dynamics of outer solar system objects, from the Kuiper Belt to the Oort cloud.

F.S. and J.K. are supported by an appointment to the NASA Postdoctoral Program at the Goddard Space Flight Center, administered by Oak Ridge Associated Universities through a contract with NASA.

\section{REFERENCES}

Anderson, M. M., Siemion, A. P. V., Barott, W. C., et al. 2011, ApJ, 744, 15 Aplin, K. L., Harrison, R. G., \& Rycroft, M. J. 2008, Space Sci. Rev., 137, 11 Atreya, S. K., Mahaffy, P. R., Niemann, H. B., Wong, M. H., \& Owen, T. C. 2003, Planet. Space Sci., 51, 105

Baines, K. H., Delitsky, M. L., Momary, T. W., et al. 2009, Planet. Space Sci., 57,1650

Balser, M., \& Wagner, C. A. 1960, Nature, 188, 638

Barth, E. L., \& Rafkin, S. C. R. 2010, Icarus, 206, 467

Béghin, C., Canu, P., Karkoschka, E., et al. 2009, Planet. Space Sci., 57, 1872

Béghin, C., Randriamboarison, O., Hamelin, M., et al. 2012, Icarus, 218, 1028
Béghin, C., Simões, F., Krasnoselskikh, V., et al. 2007, Icarus, 191, 251

Benz, W., Kallenbach, R., \& Lugmair, G. 2000, From Dust to Terrestrial Planets, Space Sciences Series of ISSI, Vol. 9 (Reprinted from Space Science Reviews, vol. 92, 432; Berlin: Springer-Verlag)

Bliokh, P. V., Nickolaenko, A. P., \& Filippov, Yu. F. 1980, Schumann Resonances in the Earth-ionosphere Cavity (Oxford: Peter Peregrinus)

Budden, K. G. 1979, Phil. Trans. R. Soc. A, 290, 405

Capone, L. A., Whitten, R. C., Prasad, S. S., \& Dubach, J. 1977, ApJ, 215, 977

Chandler, M. O., \& Waite, J. H. 1986, Geophys. Res. Lett., 13, 6

Connerney, J. E. P., Acuna, M., Ness, H., \& Norman, F. 1991, J. Geophys. Res., 96, 19023

Cooper, G., Kimmich, N., Belisle, W., et al. 2001, Nature, 414, 879

Encrenaz, T. 2008, ARA\&A, 46, 57

Farrell, W. M., Kaiser, M. L., Desch, M. D., et al. 1999, J. Geophys. Res., 104, 3795

Fegley, B., \& Prinn, R. G. 1988, ApJ, 324, 621

Fischer, G., \& Gurnett, D. A. 2011, Geophys. Res. Lett., 38, L08206

Fouchet, T., Bézard, B., \& Encrenaz, T. 2005, Space Sci. Rev., 119, 123

Fulchignoni, M., Ferri, F., Angrilli, F., et al. 2005, Nature, 438, 785

Galejs, J. 1972, Terrestrial Propagation of Long Electromagnetic Waves (New York, NY: Pergamon)

Grard, R., Hamelin, M., López-Moreno, J. J., et al. 2006, Planet. Space Sci., 54, 1124

Greifinger, C., \& Greifinger, P. 1978, Radio Sci., 13, 831

Grimalsky, V., Koshevaya, S., Kotsarenko, A., \& Enriquez, R. P. 2005, Ann. Geophys., 23, 2559

Guillot, T. 2005, Annu. Rev. Earth Planet. Sci., 33, 493

Gurnett, D. A., Morgan, D. D., Granroth, L. J., et al. 2010, Geophys. Res. Lett., 37, L17802

Hamelin, M., Béghin, C., Grard, R., et al. 2007, Planet. Space Sci., 55, 1964

Hansell, S. A., Wells, W. K., \& Hunten, D. M. 1995, Icarus, 117, 345

Hofe, R. 2005, Diploma Thesis, Graz University of Technology, Graz, Austria

Kallenbach, R., Encrenaz, T., Geiss, J., et al. 2003, Solar System History from Isotopic Signatures of Volatile Elements, Space Sciences Series of ISSI, Vol. 16 (Reprinted from Space Science Reviews, vol. 106, 444; Berlin: SpringerVerlag)

Krasnopol'sky, V. A. 1980, Kosm. Issled., 18, 429

Lewis, J. S. 1995, Physics and Chemistry of the Solar System (San Diego, CA: Academic Press)

Lindal, G. F. 1992, AJ, 103, 967

Lindal, G. F., Lyons, J. R., Sweetnam, D. N., Eshleman, V. R., \& Hinson, D. P. 1987, J. Geophys. Res., 92, 14987

Liu, J. 2006, PhD thesis, Caltech, California

Liu, J., Goldreich, P. M., \& Stevenson, D. J. 2008, Icarus, 196, 653

Lodders, K. 2004, ApJ, 611, 587

Lodders, K., \& Fegley, B. 1994, Icarus, 112, 368

López-Moreno, J. J., Molina-Cuberos, G. J., Hamelin, M., et al. 2008, Geophys. Res. Lett., 35, L22104

Lunine, J. I., \& Stevenson, D. J. 1987, Icarus, 70, 61

Madden, T., \& Thompson, W. 1965, Rev. Geophys., 3, 211

Mahaffy, P. R., Niemann, H. B., Alpert, A., et al. 2000, J. Geophys. Res., 105, 15061

Matousek, S. 2007, Acta Astronaut., 61, 932

Michael, M., Tripathi, S. N., Borucki, W. J., \& Whitten, R. C. 2009, J. Geophys. Res., 114, E04008

Michael, M., Tripathi, S. N., \& Mishra, S. K. 2008, J. Geophys. Res., 113, E07010

Molina-Cuberos, G. J., Morente, J. A., Besser, B. P., et al. 2006, Radio Sci., 41 RS1003

Mushtak, V. C., \& Williams, E. R. 2002, J. Atmos. Sol.-Terr. Phys., 64, 1989

Nellis, W. J., Weir, S. T., \& Mitchell, A. C. 1996, Science, 273, 936

Ness, N. F., Acuna, M. H., Behannon, K. W., et al. 1986, Science, 233, 85

Nickolaenko, A. P., \& Hayakawa, M. 2002, Resonances in the Earth-ionosphere Cavity (Dordrecht: Kluwer)

Pechony, O., \& Price, C. 2004, Radio Sci., 39, RS5007

Petrenko, V. F., \& Whitworth, R. W. 2002, Physics of Ice (New York, NY: Oxford Univ. Press)

Pfaff, R. F. 1996, in Modern Ionospheric Science, ed. H. Kohl et al. (Berlin: Bauer), 459

Pfaff, R. F., Rowland, D., Freudenreich, H., et al. 2010, J. Geophys. Res. Space, $115, \mathrm{~A} 12324$

Ruf, C., Renno, N. O., Kok, J. F., et al. 2009, Geophys. Res. Lett., 36, L13202

Russell, C. T., Strangeway, R. J., Daniels, J. T. M., Zhang, T. L., \& Wei, H. Y. 2010, Planet. Space Sci., 59, 965

Schumann, W. O. 1952, Z. Nat.forsch. A, 7, 149

Sentman, D. D. 1990a, J. Atmos. Terr. Phys., 52, 35 
Sentman, D. D. 1990b, Icarus, 88, 73

Sentman, D. D. 1995, in Handbook of Atmospheric Electrodynamics, ed. H. Volland (Boca Raton, FL: CRC Press), 267

Simões, F. 2007, PhD thesis, Univ. Pierre et Marie Curie, Paris

Simões, F., Berthelier, J. J., Godefroy, M., \& Yahi, S. 2009, Geophys. Res. Lett., 36, L14816

Simões, F., Grard, R., Hamelin, M., et al. 2007, Planet. Space Sci., 55, 1978

Simões, F., Grard, R., Hamelin, M., et al. 2008a, Icarus, 194, 30

Simões, F., Hamelin, M., Grard, R., et al. 2008b, J. Geophys. Res., 113, E07007

Simões, F., Pfaff, R. F., Berthelier, J.-J., \& Klenzing, J. 2011a, Space Sci. Rev.
Simões, F., Pfaff, R. F., \& Freudenreich, H. 2011b, Geophys. Res. Lett., 38 , L22101

Simões, F., Rycroft, M., Renno, N., et al. 2008c, Space Sci. Rev., 137, 455

Stevenson, D. J., \& Lunine, J. I. 1988, Icarus, 75, 146

Tobie, G., Grasset, O., Lunine, J. I., Mocquet, A., \& Sotin, C. 2005, Icarus, 175 , 496

Tsiganis, K., Gomes, R., Morbidelli, A., \& Levison, H. F. 2005, Nature, 435, 459

Tyler, G. L., Sweetnam, D. N., Anderson, J. D., et al. 1989, Science, 246, 1466

Yair, Y., Fischer, G., Simões, F., Renno, N., \& Zarka, P. 2008, Space Sci. Rev., 137,29 